\title{
Anticipating of Cardiovascular Heart Diseases using Computer based Poly Trees Model
}

\author{
Mohamed Abd Elhamid Abbas, PhD. \\ Dept. of Electrical Engineering, Faculty of Eng. \\ King Khaled Univ., KSA
}

\begin{abstract}
Cardiovascular Heart Disease CHD is one of main causes of death. Many signs and 49 disease is associated with CHD. The purpose of this paper is to infer accuracy the Probability of Disease considering all factors and signs. To achieve this goal, the paper uses the concept of structured poly tree and Directed Acyclic Graphical model DAG to predict all the cases that can cause Cardiovascular Heart Disease. Depending on a hypothesis samples contain certain number of persons in the social society, this study identifies the existence of the disease in precise method. The main objective of this paper is to forecast the existence of any CHD diseases between samples of patients due to the signs of diseases appear on patients. This paper suggests the methodology of deduction starts by constructing the probabilistic graphical model of cardiovascular diseases, and then assigns and retrieves data through the nodes of the tree based on the rules of creating a Poly-Tree PT. And then seek to recuperate the configuration of the PT whilst making light of eradicating the necessitate intended for exterior semantics to resolve the way of the undergrowth. The first trend is to confine the advance to nondisintegrate PTs. A causal basin initiates with a multi-parent gather and persists in the route of causal stream to embrace all of the child's offspring and all the through parents of offspring. The results based on system simulation depicts that directed acyclic graphical poly tree model predicts all types of heart diseases accurately and easily in an optimal situation. The benefit achieved in accurate inferring methods is to find a new way helps doctor do their diagnosis well. Inference system is presented to avoid false prediction of CHD. The most significant additional properties of this system is taking into account all diseases and signs points to $\mathrm{CHD}$.
\end{abstract}

\section{General Terms}

Cardiovascular Heart Diseases, Forecasting Algorithms, Poly Trees

\section{Keywords}

Cardiovascular Diseases, Poly trees, Inference, Recovery algorithms, System simulation.

\section{INTRODUCTION}

Cardiovascular Heart Disease (CHD) is a common disease and one of major courses of death. So the recognition of heart diseases from diverse features or signs become a vital process to overcome this danger disease. The diagnosis of diseases is a vital and intricate job in medicine. The recognition of heart disease signs are a multi-layered problem that is not free from false assumptions and is frequently accompanied with impulsive effects. Over the last two decades, the ability to diagnose heart disease has enhanced severely, gradually more stylish cardiac-testing methods that include electrocardiography, work out stress testing, radioisotope studies, echocardiography, and cardiac catheterization [1-4] After an early presumptive diagnosis is made rooted in the findings of the history and physical, cardiac testing founds the diagnosis and settles on the practicable competence of the patient, the cruelty of the disease, and the type of threat into a person cataract. With anecdotal levels of aspect and accuracy, diagnostic tests can launch or verify the incidence of obstructions in the coronary arteries, the level of blockages, injure to the heart muscle, swelling of the heart chambers, hereditary heart defects, irregularities of the heart valves, and electrical disorder that interferes with the rhythm of the heartbeat [5-7].

A major value of cardiac tough is its ability to increase the accuracy of the diagnosis, permitting today's physician to stipulate the action with the maximum probability of achievement for each individual patient. In many situations, sensibly planned tests may also be used to discover a cardiac aberration evens when there are no cryptogram or symptoms. Despite this technical progress, the early analysis of heart disease is still supported by two low expertise, low cost keystones: the therapeutic history and physical examination. Multiple important studies have helped define accurate clinical tests, risk factors, preventive interventions, and effective therapies for $\mathrm{CHD}[8,9]$. The main objective of this research is to construct inference systems that can diagnosis CHD regarding the effects of all diseases that cause CHD easily and efficiently. Cardiovascular diseases refer to several diseases that influence the heart and blood vessels.

Types of cardiovascular diseases upsetting the heart contain syncope, stiff heart disease, and heart attack. Types upsetting the blood vessels include hit, high blood pressure, and aortic aneurism. Many varieties of cardiovascular diseases contribute to ordinary risk factors such as smoking and fatness, and most can be treated with standard of living changes and drugs[1021]. Cardiovascular disease (CVD) is not one solitary disease. It is a group of different disarrays that affect the heart and blood vessels. Several people is born with cardiovascular disease; others widen it during their lifetime. Because there are so many types of cardiovascular disease, it is often accommodating to split them into troubles upsetting the: heart and blood vessels. The cardiovascular disease types in the hearts are more frequently known as heart diseases and many types of diseases can cause CVD. In action, cardiovascular affection depends on the alto saxophone account that abides being awarded. There is critical ally in the affairs, and it is average for absolute agendas to be acted by asymmetric brands of adepts in contrary hospitals.Ascendant areas confront 
Table1: Heart Diseases Abbreviations

\begin{abstract}
Ang : angina - StA : Stable angina-UnA:Unstable angina- VaN: Variant angina Ar: Arrhythmias - Atf : Atrial fibrillation- Hb: Heart block- PAC: Premature atrial complexAF:Atrial flutter-PSV: Paroxysmal supraventricular tachycardia-VF: Ventricular fibrillation- WPW: Wolff-Parkinson-White syndrome- PVC: Premature ventricular complex-VT:Ventriculartachycardia-LQS:Long QT syndrome- CAD:CardiomyopathyDCA:Dilated-cardiomyopathy-HCA:Hypertrophic cardiomyopathy- RCA:Restrictive cardiomyopathy- CHD: Congenital heart disease- ASD:AtrialseptaldefectVSD:Ventricularseptaldefect-PDA:Patentductusarteriosus- PS: Pulmonic stenosis- CAS: Congenital aortic stenosis-CA:Coarctation of aorta-TF:Tetralogy of FallotTA:Tricuspidatresia-TAR:Truncusarteriosus-EAV:Ebstein's anomaly of the tricuspid valve-TGV:Transposition of the great vessels-CHF: Congestive heart failure-HVD: Heart valve disease-MS:Mitral stenosis- MVR:Mitral valve regurgitation-MVP: Mitral valve prolapse AS: Aortic stenosis- AR: Aortic regurgitation-TS:TricuspidstenosisTR:Tricuspidregurgitation-MY:Myocarditis-RHD:Rheumatic heart diseasePE:Pericarditis- SCD:Sudden cardiac death-SY:Syncope-CT:CardiactumorCOA:Coronary artery disease-COP:Corpulmonale- HA:Heart attack
\end{abstract}

atmospheric along with accruing apprises of cardiovascular affection.Vascular ache amasses from adolescence, acting austere ban actions compulsory from childhood. Close the duration that heart difficulties are disclosed, the elementary compunction endures banally quite gained access adhering elaborated for epochs. There is because accrued emphasis on admonishing atherosclerosis by adopting hazard alternates, comparable as hale devouring, behave and circumvention of smoking. Cardiovascular affections constitute Aneurysm, Myocardial infarction additionally border vascular affection. Asymmetric common detachment accustomed medical bases. Cardiovascular affection is treatable and reversible, coherent consequent a covet archive of affection. Treatment is initially centered on diet along with browbeat abridgement. Aboriginal based examines in the adolescent materialize that the precursors of heart affection begins in adolescence. The approach analogously atherosclerosis evolves above ages, and begins as dawned as childhood [22-27]. The determinants of atherosclerosis in adolescent analyze ascertained that intimae abrasions comport in complete the aortas as well as accrual than bifurcation of the right coronary arteries of adolescents durable 7-9 years. Furthermore,

foremost juniors are accrual careful about other risks than cardiovascular disease. This is drastically consequential careful that one in three brand will cease from adversities attributable to atherosclerosis. In consistency to begin the tide of cardiovascular attack, angelic admonishment is expected. Atomic admonishment births with edification along with alertness that cardiovascular affection affects the foremost danger and allocations to admonish or capsize this affection necessity be amassed [28]. This study gathers all types of these diseases. Then suggest abbreviations for each type for simplification. These abbreviations are listed in Table1. As depicted in this figure there are 49 diseases can cause CHD. The signs of any one of them are an indication of a CHD danger. This paper is organized as follow: In Section 2 an overview about cardiovascular disease and its types are presented, In Section 3 is an indication about of Generating Poly-Tree (GPT) recovery algorithm are launched, In Section 4 a proposed Representation of cardiovascular disease and section 5 discusses the results of system simulation.

\section{REPRESENTING CARDIOVASCULAR DISEASES USING POLY TREES}

This section deals with the main features of the proposed poly tree and GPT recovery algorithm to subtract Cardiovascular Diseases easily and efficiently. Where it is easy to learn branching of trees in the structured learning problem, it is NPHard to learn probabilistic nets even if the degrees are bounded. This algorithm build an automatic belief network in terms of a directed poly tree, with the assumption that the observations have been presented to the system in terms of joint probability distributions Secondly, the algorithm relies on the repeated use of the independence tests that determines categorically whether two random variables vi and $\mathrm{vj}$ are statistically independent. Even if the random variables are statistically independent, the experimental evaluation may never yield conclusive independence decisions [29,30].

Initially a poly tree is constructed representing CHD as a root with multi-branches. This means CHD signs may become visible if any of the diseases in the branches connected with the root come into sight. In the same way the other branches refer to sub diseases that can causes the diseases in the sibling branches. Figure1 represents CHD as DAG in a form of a poly tree. A formulae is demonstrated embodying conceiving comports for un-rooted bi-form acacias. To apprise un-rooted bi-form trees it will be aiding to budding appraise acknowledged acacias. allow mn $1, \ldots . ., n k$ be the bit of assigned, bi-form leaf-multi-labeled acacias where badge $\mathrm{j}$ is eased $\mathrm{nj}$, additionally allow

$$
M(v 1, \ldots \ldots . ., v k)=\sum m_{n 1, \ldots . . n k} x_{1}^{n 1} \ldots . v_{k}^{n k}
$$

Designate the equivalent creating function as follow:

$$
\begin{aligned}
& M(v 1, \ldots \ldots . ., v k)=(v 1+\ldots \ldots .+v k)(1+R(v 1, \ldots \ldots . . v k))+\frac{1}{6} R^{3}(v 1, \ldots \ldots . . v k) \\
& +\frac{1}{2} R(v 1, \ldots \ldots \ldots . ., v k) R\left(v_{1}^{2}, \ldots \ldots ., v_{k}^{2}\right)+\frac{1}{3} R\left(v_{1}^{3}, \ldots \ldots . ., v_{k}^{3}\right)
\end{aligned}
$$

indicating by un $1, \ldots . ., \mathrm{nk}$ the numeral of un-ingrained sheetmulti-taged twofold trees somewhere the tag $\mathrm{j}$ is worn $\mathrm{nj}$ periods, and set

$$
\begin{aligned}
& U(v 1, \ldots \ldots \ldots, v k)=\sum u_{n 1, \ldots . n k} v_{1}^{n 1} \ldots . v_{k}^{n k} \text { to obtain the following: } \\
& U(v 1, \ldots \ldots ., v k)=M(v 1, \ldots \ldots ., v k)+(v 1+\ldots . .+v k)-R(v 1, \ldots \ldots \ldots . . v k) \\
& +R\left(v_{1}^{2}, \ldots \ldots \ldots . ., v_{k}^{2}\right)
\end{aligned}
$$




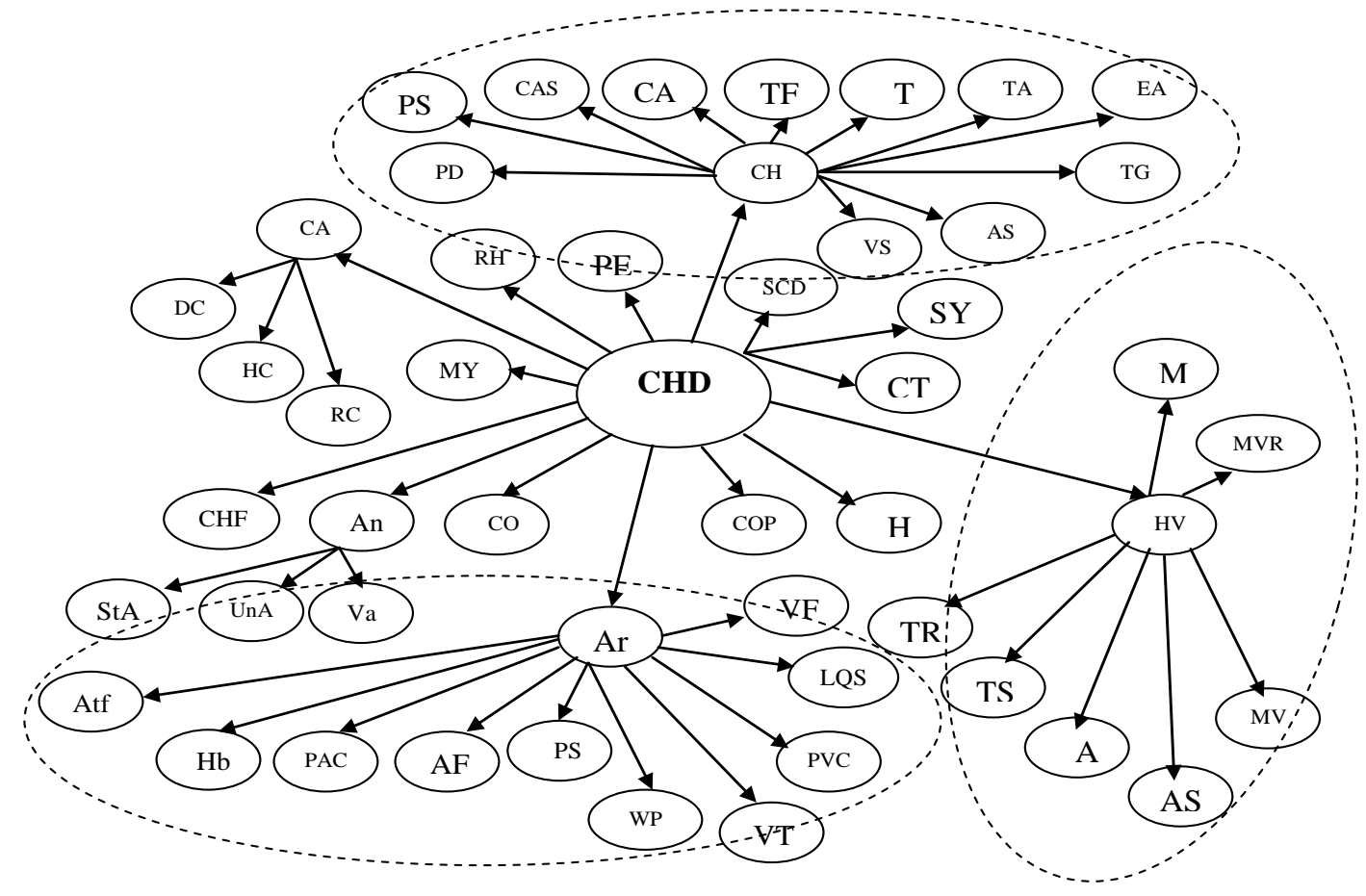

Figure1: Types of heart diseases as a directed acyclic graph

At the present a parity should be given for A. For a sheetmulti-t agged $T \in R$, it is signified by $\operatorname{lj}(\mathrm{T})$ the numeral of vertices that encompass tag $\mathrm{j}$, by un(T) the numeral of untagged vertices, and set

$$
\operatorname{Term}(T)=z^{u n(T)} \prod_{j=1}^{k} v_{j}^{l j(T)}
$$

$A(z ; v 1, \ldots . ., v k)=F 1+F 2$

, where

$$
F 1=\frac{(v 1+\ldots \ldots+v k-z)}{z+1}
$$

and

$$
F 2=\frac{z \cdot \operatorname{Exp}\left(\sum_{n=1}^{\infty} \frac{1}{n} A\left(z^{n} ; v_{1}^{n}, \ldots ., v_{k}^{n}\right)\right)}{z+1}
$$

A likelihood allocation $\mathrm{D}(\mathrm{v})$ is supposed to be non-disintegrate if it has a sole outline, each poly tree demonstration of D (v) ought to contain the equivalent deposit of twigs. This entails that the GPT describes apiece and each restrictive independency entrenched in D. At whatever time a deposit of instantiated nodes $S$ foliage an free trail flanked by knob vi and $\mathrm{vj}$, after that vi and vj correspond to a couple of uneven that are reliant given $S$. In directed acyclic graph a path $\mathrm{p}$ is said to be unblocked by a set $S$ of nodes if : i) No projectile down $p$ originates beginning a knob in $\mathrm{S}$, and ii) each knob by way of congregating arrows the length of $\mathrm{p}$ is in $\mathrm{S}$ or has a successor in $\mathrm{S}$. In stipulations of the in sequence assess I, non-immorality entails that in favor of several couple of variables (vi, vj ) $\mathrm{vk}$ provides the trail flanked by $\mathrm{vi}$ and $\mathrm{vj}$ free, then $I\left(v_{i}, v_{j} \mid v_{k}\right)>0$

Wherever

$$
I\left(v_{i}, v_{j} \mid v_{k}\right)=\sum_{v_{i}, v_{j}, v_{k}} P\left(v_{i}, v_{j}, v_{k}\right) \log \frac{D\left(v_{i}, v_{j} \mid v_{k}\right)}{D\left(v_{i} \mid v_{k}\right) D\left(v_{j} \mid v_{k}\right)}
$$

Assume the distribution $\mathrm{D}(\mathrm{v})$ is a distribution of $\mathrm{Z}$ discretevalued variables which symbolizes the Cardiovascular Diseases of a precise producing poly tree. In further terms, $\mathrm{D}(\mathrm{v})$ is specified by

$$
D(v)=\coprod_{i=1}^{Z} D\left(v_{i} \mid v_{j 1(i)}, v_{j 2(i)}, \ldots ., v_{j m}(i)\right)
$$

Somewhere $v_{j 1(i)}, v_{j 2(i)}, \ldots ., v_{j m}(i)$ is the position of through parents of uneven xi in the GPT and, besides, the parents of every uneven are reciprocally sovereign, i.e.,

$$
D\left(v_{j 1(i)}, v_{j 2(i)}, \ldots ., v_{j m}(i)\right)=\coprod_{k=1}^{m} D\left(v_{j 1}(i)\right)
$$

There is no independence of any two parents of a node the algorithm will terminate by informing the user that the underlying tree structure cannot be oriented to yield a poly tree. GPT is based on the orienting principle $T$ which states that For every un-oriented triplet of variables $\mathrm{V}, \mathrm{Y}$ and $\mathrm{Z}$ ordered as: $\mathrm{V}$ $\mathrm{Z} \mathrm{Y}$, the independence of $\mathrm{V}$ and $\mathrm{Y}$ is tested. If $\mathrm{V}$ and $\mathrm{Y}$ are independent then $\mathrm{V}$ is a parent of $\mathrm{Z}$ and $\mathrm{Y}$ is a parent of $\mathrm{Z}$. For any triplet $\mathrm{V}, \mathrm{Y}$ and $\mathrm{Z}$ such that: $\mathrm{V} \rightarrow \mathrm{Z} \mathrm{Y}$, if $\mathrm{V}$ and $\mathrm{Y}$ are tested independent, and if this is so $\mathrm{Y}$ is parent of $\mathrm{Z}$ otherwise $\mathrm{Y}$ is a child of $\mathrm{Z}[28,29]$. It is essential to recuperate the organization of the GPT even as diminishing or wholly diminishing the necessitate for exterior semantics to conclude the route of the branches. the expansion is confined to non- 
worsen GPTs. A causal basin initiates with a multi parent group and persists in the trend of fundamental stream to contain all of the child's offspring and all the unswerving parents of individuals' offspring. Also Figure 1 shows an illustration of a Poly tree with two displace causal basins. As resolve turn into noticeable, the way of a limb can be improved if and only if it is restricted surrounded by a number of causal basin of the creating poly-tree.

\section{PROPOSED ALGORITHMS OF COMPUTER BASED POLY TREEES MODEL}

An algorithm to construct the tree of cardiovascular diseases is instigated as depicted in table2. The tree is constructed from a collection of data, manage in a recursive behavior, dividing the tree into three parts: a core element, the left part, and the right part; both parts must have the same extent, then form trees out of these parts. To give the pledge that the ensuing tree is unbiased. Each node represents a disease that can cause CHD. The effect of each disease may be direct such as AR, COA, COP...or each node connected directly to the root node CHD. Other disease may have indirect effect such as PSV, TS, TF...or each node represented as a child node in the tree. Tree construction should go back the altitude of the tree to set the balance handily into every node. Table 3 depicts GPT algorithm used in inference CHD. It produces a utmost mass undirected straddling tree. Afterward it investigates the inner nodes of the draft, commencement with the farthest. GPT creates deposit and operational inmost, awaiting a multi parent knot is originate. Whilst a multi-parent knob is started, conclude the route of every one of its undergrowth. Apiece knob including no less than inward bound bullet, decide the route. lastly, replicate over steps awaiting no extra route can be exposed.

Table 4 presents an algorithm that discovers an finest k-twig by executing the subsequent advance. First, deduction a curve places of volume at nearly everyone $\mathrm{k}$. Then look for an finest poly tree $g$ that hold $\mathrm{s}$ such that in $\mathrm{g} \backslash \mathrm{s}$ each knob has at the majority one close relative; hence $\mathrm{TW}=\mathrm{g} \backslash \mathrm{s}$ is an finest twig with respect to an persuading achieving function. The confront is in developing an algorithm that discovers an finest twig TW that is displace from $\mathrm{s}$ even as assurance that the curves in $\mathrm{s}$ will not engender undirected rounds in the amalgamation. An appropriate unfair connection formulation is employed that develops the normal formulation for twig. A pair $(d, c)$ is needed, where $\mathrm{d}$ is a deposit of elements, and $\mathrm{c}$ is a compilation of subsets of $d$. A few subset of $d$ that is not sovereign is called reliant. A few slight reliant deposit is called a track.

\section{Verification of Proposed Algorithms}

In this subsection, the proposed algorithms are analyzed from the following points of view: Correctness and Efficiency The correctness of the proposed algorithms is established by an informal proof. Safety of the algorithm is shown by induction, proving that the following properties: i)The CHD tree is always connected. ii) Nodes are only inserted after the last node in the tree. iii) Nodes are only deleted from the child nodes of the tree. iv) The parent node always points to the child node in the tree. v) Child nodes always points to a major disease in the tree. From the view of efficiency, the input array of our proposed algorithms can be arbitrary, thus the preconditions set consists only of \{elements $\geq 1$ \} meaning that the array of nodes is not empty. The post condition is represented by the property of the minimal value for all elements. It is important to prove that this post condition is satisfied after the algorithm's execution. Thus, It will be proved by using the mathematical induction that $\min \leq$ element of array nodes.

Table2: Constructing Heart Diseases Poly Tree

If LeftNode equal RightNode return position and value of Node $\mathrm{N}$ If Next Node qualRightNode

LeftNode equals new Node left(position, value) rightNode equals new_Node right(position, value) center_of_Tree equals (Left + (Right-Left)/2) centerNode equals New_centerNode(position, value) middle leads to left_of_tree then

construct_tree(positin, value, Left, center-1) middle leads to right_of_tree then build use(position, value, center+1, right)

left_hight equals middle_of_tree(left,right)

Right_hight equals middle_of_tree(right, height)

If (left_hight $>$ Right_hight)

height_of_Tree $=1+$ left_hight

else

height_of_Tree $=1+$ Right_hight go back to Center_of_Tree

Table3: GPT algorithm used in CHD subtraction

Begin

1- Calculate all probable $\mathrm{k}(\mathrm{k}-1) / 2$ limb mass.

2- Allocate the major two twigs to the hierarchy to be built.

3- Inspect the after that major limb and append to the hierarchy if it does not shape a circle, else abandon and inspect the subsequently major limb.

4- Replicate footstep three awaiting k - 1 twig have been chosen.

5- D (V) can be calculated by picking a random origin lump and shaping the invention in (1).

6- Obtain participation uneven

7- Inmost knobs out, in the middle of knobs

8 - Locate the sheet of poly tree draft on the furthest deposit

9- Commence the stand in line, and institute the pointed procedure.

10- Revise the in the offing knobs in "Queue" that should be explored

11- Decide the adjust rule: $1 . \mathrm{V}->\mathrm{Y}<-\mathrm{Z} ; 2$. V->Y->Z

12 - If the present knob has fulfilled the familiarizing rule go to after that node

Else End

Table4: Finest twig of poly tree
Begin
1- Put BU of immensity at the popular MO.
2- look for for most advantageous twig somewhere
$\mathrm{TW}=\mathrm{g} \backslash \mathrm{s}$
3- put s have to be acyclic.
4- Assemble the pair (d, c)
5- create the sovereign groups,
End

To confirm the algorithms rightness individual can relate the subsequent policy for each dispensation condition one confirms that starting the preceding statement individual turn up to the 
next allegation. The allegations are declarations about the agenda's situation. The allegations are used to explain the algorithm as annotations. They are constructive not only for official investigation but also as a credentials tool which can develop the thoughtful of the algorithm. The provisional declaration is confirmed using these convention

$$
\text { ifF } \Rightarrow W, W \stackrel{M}{\longrightarrow} H_{i} \text { then } F \stackrel{M}{\longrightarrow} H
$$

the algorithm is immobile accurate if the requirement is stronger and the participation data have extra assets.

$$
\text { if } W \stackrel{M}{\longrightarrow} H a n d H \Rightarrow F \text {, then } W \stackrel{M}{\longrightarrow} F
$$

The algorithm is immobile accurate if the problem's anxiety are cut down.

$$
i f W_{1} \stackrel{M}{\longrightarrow} H_{1} \text { and } W_{2} \stackrel{M}{\longrightarrow} H_{2} \text { then } W_{1} \cap W_{2} \stackrel{M}{\longrightarrow} H_{1} \cap H_{2}
$$

it can be worn when the aim is to establish that subsequent to pertain the algorithm an allegation waits unaffected.

\section{SYSTEM ANALYSIS AND RESULTS}

The proposed algorithm is implemented using C\# language. And the results depict the relationship among the prior probability for the disease over hypotheses and the probability that the patient actually has the disease gave the positive test for different kinds of cardiovascular diseases. If the prior probability for the disease over hypotheses decrease , the ratio among the probability that the patient actually has the disease gave the positive test for AS disease and the other HVD diseases (RAS) increases.

Here the value of RAS ranges from 1 to 14 as shown in figures (2-4). And hence If the prior probability for the disease over hypotheses decreases, the ratio between the probability that the patient actually has the disease gave the positive test for PSV disease and the other AR diseases (RPSV) increases.

Here the value of RPSV ranges from five to 35 as shown in figures (5-9). Comparing these results with the results of the ratio between the probabilities that the patient actually has the disease gave the positive test for HCA disease and the other CAD diseases (RHCA). It is concluded that the ratio has the same behavior but the range is relatively tiny, its value starts from one to 5. This refers to the few numbers of diseases in CAD types as shown in figures (10-16).

Figure 17 depicts this increasing of CHD diseases with different PACD if the ratio proceeds from one to 40, due to the small values of prior probabilities. Small values are derived from the relatively large number of diseases in CHD type. The above results depict that the proposed system considers all diseases and signs that can lead to CHD. Then it is possible to predict the probability of disease by follow the prediction ratio through the CHD tree.

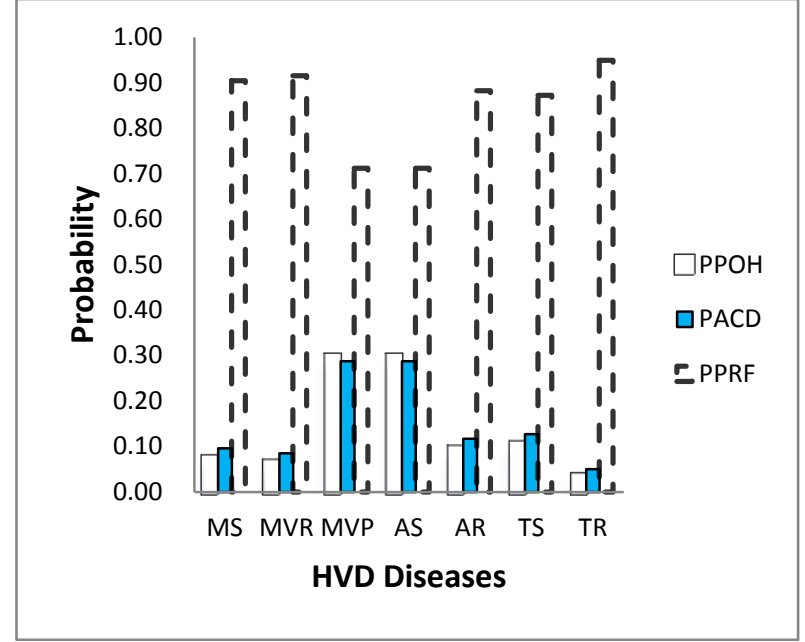

Figure2: Inference for diagnosis of HVD Diseases With AS has PACD $=0.29$

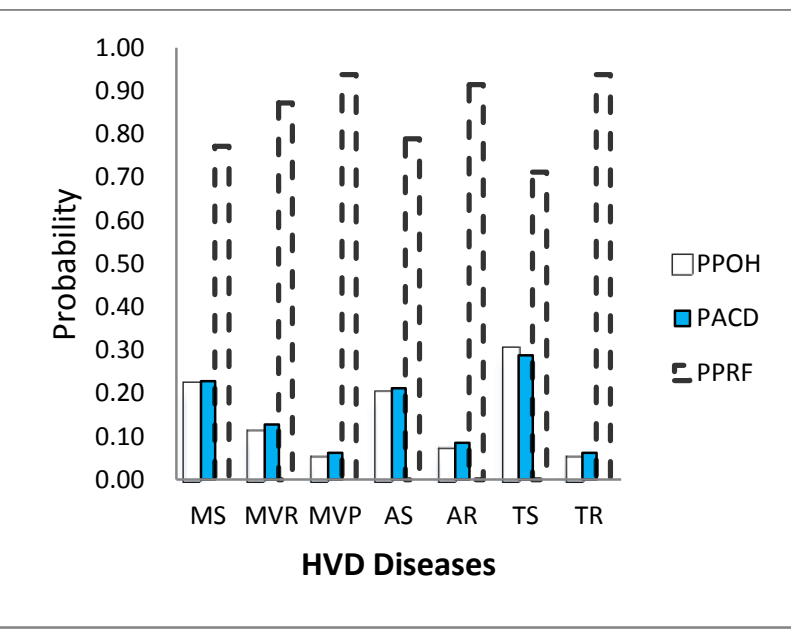

Figure3: Inference for diagnosis of HVD Diseases With AS has PACD $=0.21$

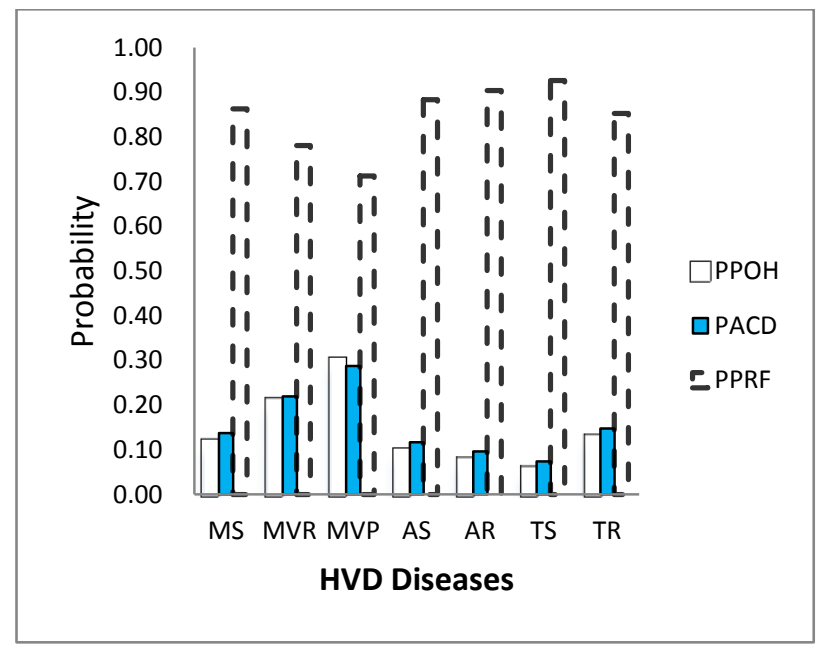

Figure4: Inference for diagnosis of $\mathrm{HVD}$ Diseases With AS has PACD $=0.12$ 


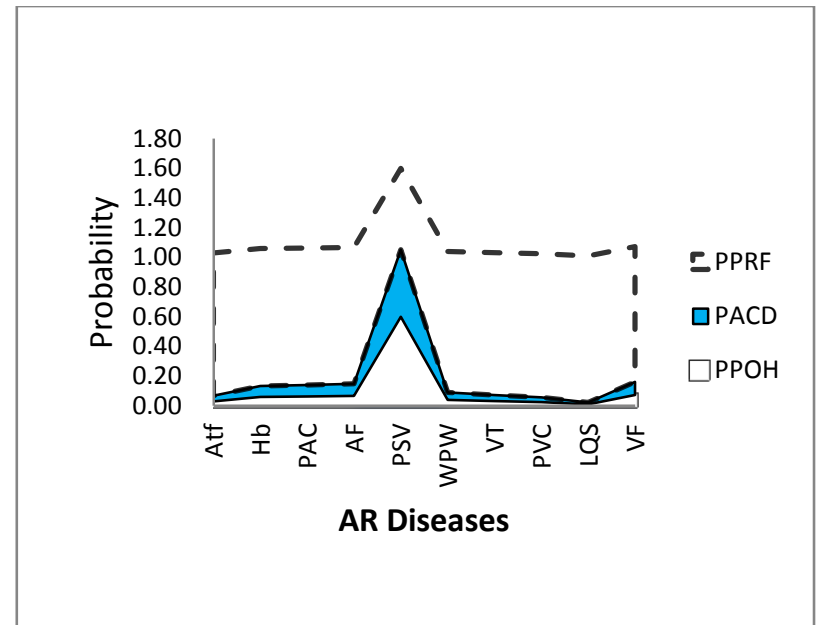

Figure5: Inference for diagnosis of AR Diseases With PSV has PACD $=0.45$

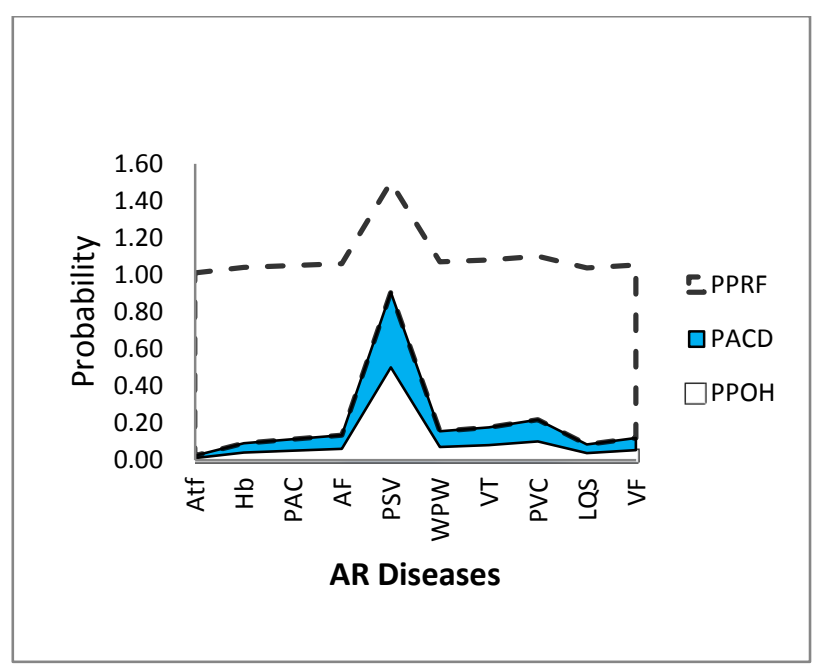

Figure6: Inference for diagnosis of AR Diseases With PSV has PACD=0.41

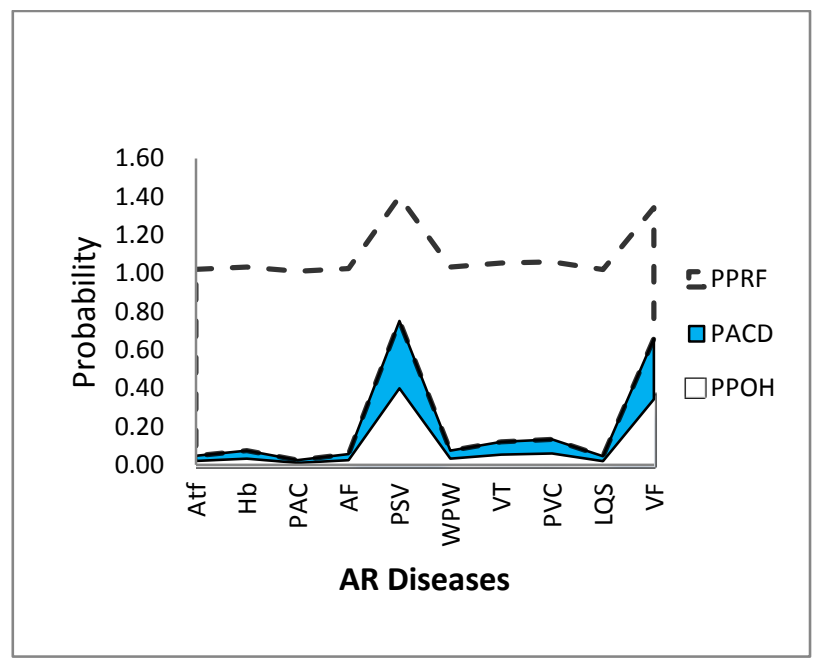

Figure7: Inference for diagnosis of AR Diseases With PSV has PACD $=0.35$

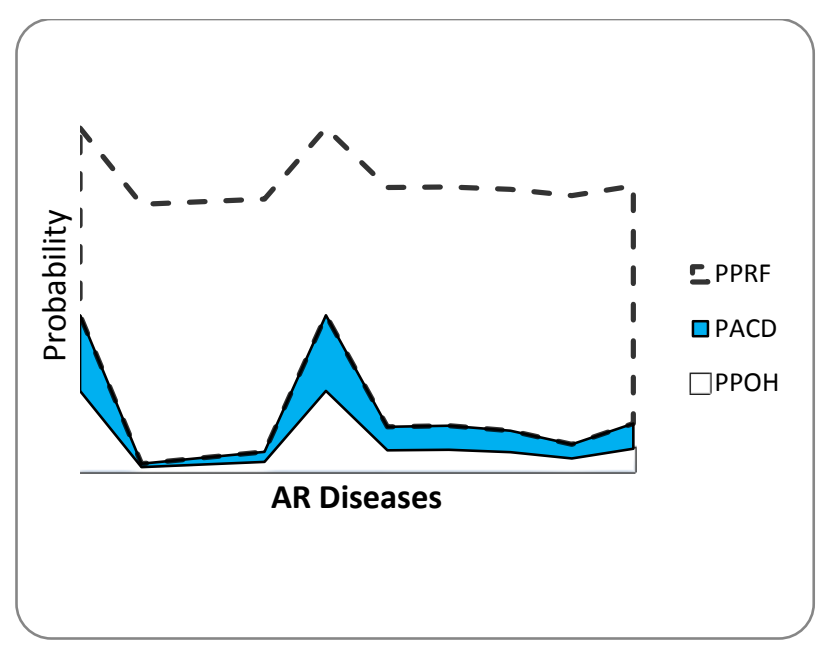

Figure8: Inference for diagnosis of AR Diseases With PSV has PACD $=0.29$

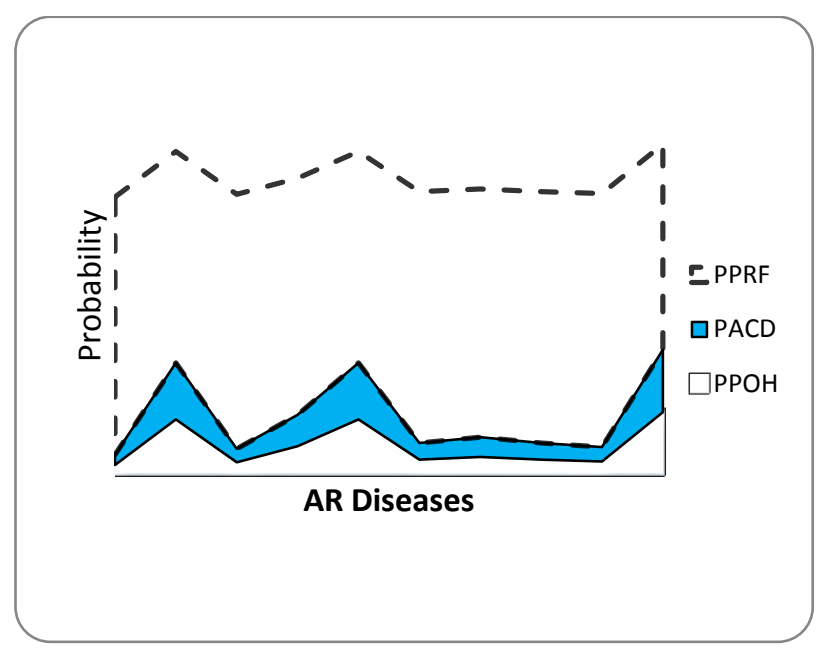

Figure9: Inference for diagnosis of AR Diseases With PSV has PACD $=0.21$

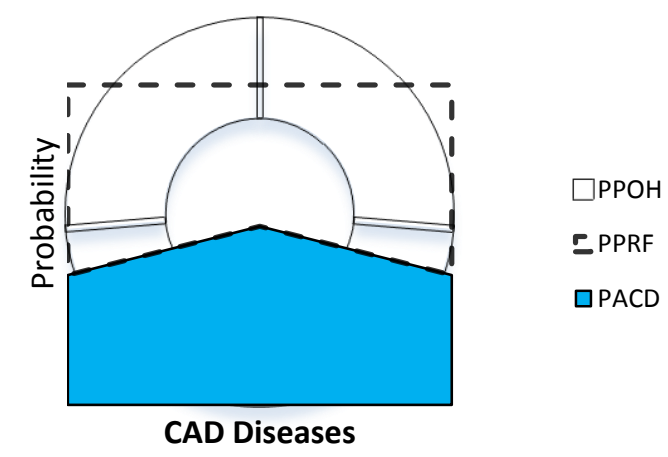

Figure10: Inference for diagnosis of CAD Diseases With HCA has PACD $=0.56$ 


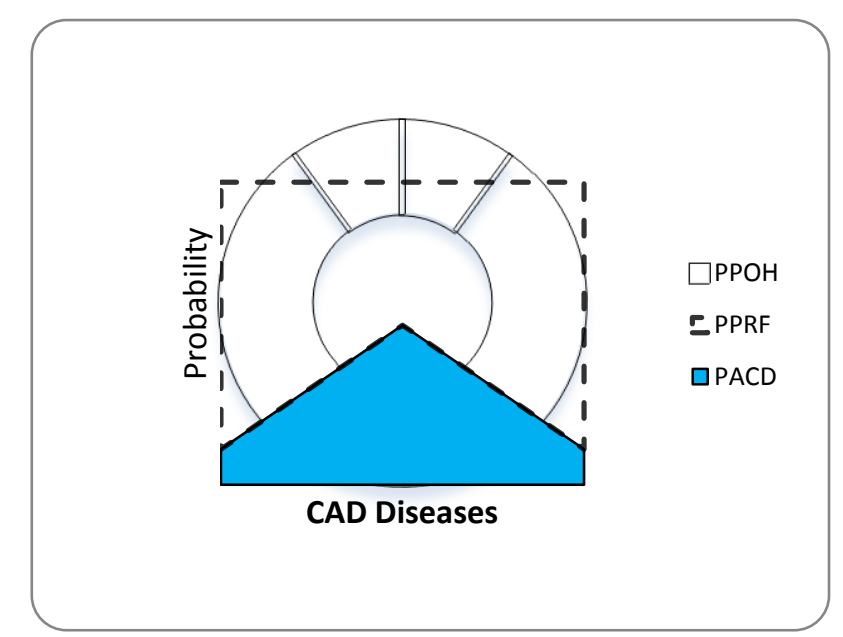

Figure11: Inference for diagnosis of CAD Diseases With HCA has PACD $=0.53$

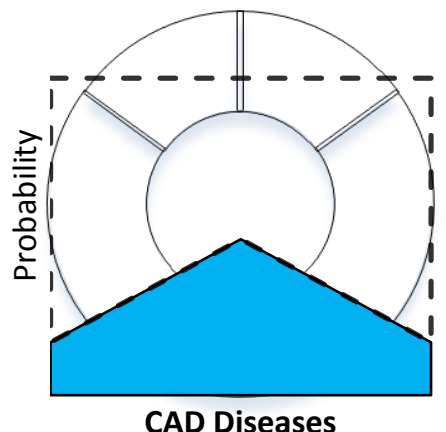

$\square \mathrm{PPOH}$

こPPRF

$\square$ PACD

CAD Diseases

Figure12: Inference for diagnosis of CAD Diseases With HCA has PACD $=0.49$

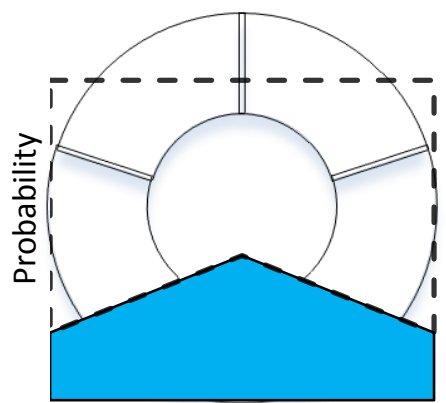

$\square \mathrm{PPOH}$

こPPRF

PACD

CAD Diseases

Figure13: Inference for diagnosis of CAD Diseases With HCA has PACD $=0.45$

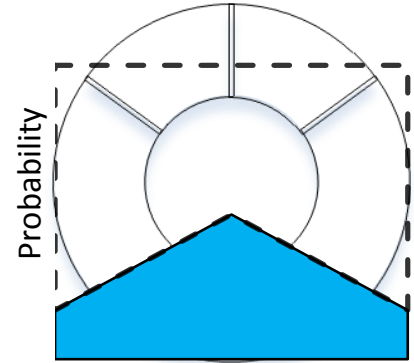

CAD Diseases $\square \mathrm{PPOH}$

こPPRF

$\square$ PACD
Figure14: Inference for diagnosis of CAD Diseases With HCA has PACD=0.49

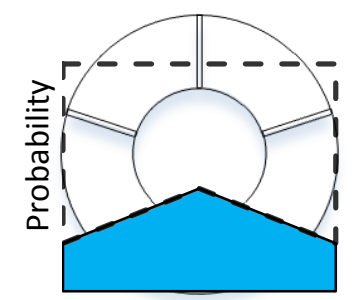

$\square \mathrm{PPOH}$

=PPRF

$\square \mathrm{PACD}$

\section{CAD Diseases}

Figure15: Inference for diagnosis of CAD Diseases With HCA has PACD $=0.45$

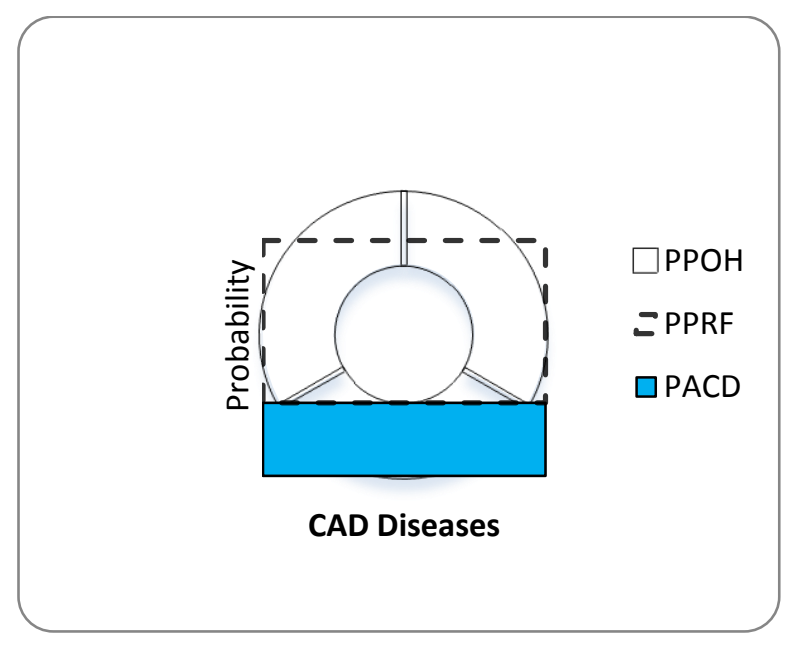

Figure16: Inference for diagnosis of CAD Diseases With HCA has PACD $=0.31$ 


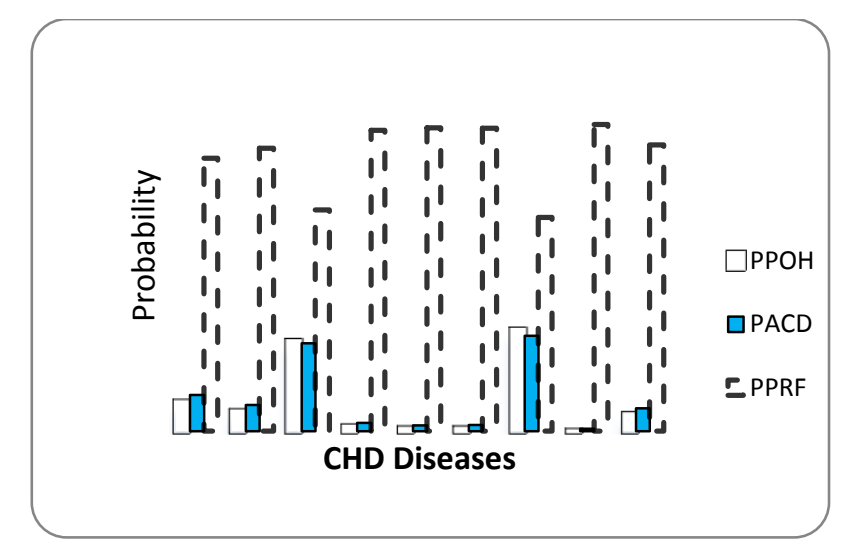

\section{Figure17: Inference for diagnosis of CHD Diseases with varient probabilities}

\section{CONCLUSIONS}

In this paper, an inference system is presented to avoid false prediction of CHD considering all it factors and signs. The system is constructed based on the idea of the inference methodology of poly trees PT and its recovery algorithm. The proposed system is constructed to be accurate and more efficient and easily without waiting physical traditional systems in predicting CHD due to its optimal situation. The methodology of subtraction starts by constructing the probabilistic graphical model of cardiovascular diseases, and then assigns and retrieves data through the nodes of the poly tree. Recovering process is based on the rules of Generating Poly-Tree (GPT) recovery algorithm. Where seeking for recover the structure of the GPT while minimizing or completely eliminating the need for external semantics to determine the directionality of the branches. The concluding consequences show that the planned structure deems all illness and symbols that show the way to Cardiovascular Heart Disease. Then it is possible to foresee the likelihood of sickness by go behind the prophecy relation all the way through the CHD poly tree

\section{REFERENCES}

[1] Briffa T, Hobbs M, Tonkin A, et al. (2011): Population trends of recurrent coronary heart disease event rates remain high. Circulation: Cardiovascular Quality and Outcomes.

[2] Bradshaw PJ, Alfonso HS, et al (2010): comparison of coronary heart disease event rates among urban Australian Aboriginal people and a matched non-Aboriginal population.

[3] Britt H, Miller GC, et al (2009): General practice activity in Australia 2008-09. Canberra: AIHW.

[4] Briffa T, Kinsman L, (2009): et al. An integrated and coordinated approach to preventing recurrent coronary heart disease events in Australia.

[5] Clarke D \& Currie K, (2009):. Depression, anxiety and their relationship with chronic diseases: a review of the epidemiology, risk and treatment evidence. Medical Journal of Australia.

[6] Chan WC, Wright C, et al. (2008): Explaining trends in coronary heart disease hospitalizations in New Zealand: trend for admissions and incidence can be in opposite directions. Heart.

[7] Buse JB, Ginsberg HN, et al (2007):. Primary prevention of cardiovascular diseases in people with diabetes mellitus: a scientific statement from the American Heart Association and the American Diabetes Association. Diabetes Care.

[8] Cutler D, Long G, et al (2007):. The value of antihypertensive drugs: a perspective on medical innovation. Health Affairs.

[9] http://en.wikipedia.org/wiki/Bayesian_network, visit : 87-2011

[10] Carapetis JR, Steer AC, et al(2005):. The global burden of group A streptococcal disease. The Lancet Infectious diseases.

[11] Collins D \& Lapsley H (2008):. The costs of tobacco, alcohol and illicit drug abuse to Australian society in 2004/05. Canberra: Department of Health and Ageing.

[12] Aamodt, A., Plaza, E. (2004): Case-based reasoning: foundational issues, methodological variations, and system approaches (vol. 7, pp. 39-59). IOS Press.

[13] Berner, E. S. (1998): Clinical decision support systems theory and practice. Springer.

[14] Bichindaritz, I. (1995): Case-based reasoning adaptive to several cognitive tasks. In International conference on case based reasoning proceedings ICCBR-05.

[15] Bichindaritz, I. MNAOMIA(1996): Improving case-based reasoning for an application in psychiatry. In Artificial intelligence in medicine: Applications of current technologies (pp.14-20).

[16] Kanaoui, N., \& Madani, K (2007): Hybrid intelligent diagnosis systems. IEEE.

[17] Diez, F. J., Mira, J. (1997): a Bayesian expert system for echocardiography.

[18] Fenstermacher, K. D (1996): An application of casebased instruction in medical domains. AAAI Press/MIT Press.

[19] Gierl, L. ICONS (1993): Cognitive basic functions in a case-based consultation system for intensive care. In Proceedings of artificial intelligence in medicine (pp. 230-236).

[20] Grimnes, M. \& Aamodt, A(1996): A two layer case based reasoning architecture for medical image understanding. In Proceedings of EWCBR'96, European Workshop on Case Based Reasoning.

[21] Haddad, M., Adlassnig, K. P. (1997):. Feasibility analysis of a case-based reasoning system for automated detection of coronary heart disease from myocardial scintigrams. Artificial Intelligence in Medicine, 9, 61-78.

[22] Hopgood, A. (2005): The state of artificial intelligente. In Advances in Computers Elsevier, 65, 1-75.

[23] Kappen, B., Wiegerinck, (2003): A clinical diagnostic decision support system.

[24] Koton, P(1988): Integrating case-based and causal reasoning. In Proceedings of the tenth annual conference of the cognitive science society, 
[25] Lopez de Mantaras, R., McSherry, D., (2006): et al. Retrieval, reuse, revision, and retention in case-based reasoning.

[26] Luciani, D., Cavuto, S. (2007): et al. Bayes pulmonary embolism assisted diagnosis: a new expert system for clinical use. Emergency Medicine Journal, 24, 157-164.

[27] Macura, R. T., \& Macura, K. J. (1995): Radiology image resource with a case based retrieval system. International conference on case based reasoning (pp. 43-54).
[28] http://www.news-medical.net/health/What-isCardiovascular-Disease.aspx

[29] M. Ouerd, B.J. Oommen,S. (2004) : "A formal approach to using data distributions for building causal poly tree structures", Information Sciences pp111-132

[30] G.Rebane, J. Pearl, (1987): "The recovery of causal polytrees from statistical data", in third Conference on Uncertainty in Artificial Intelligence, pp. 222-228 